\title{
Stochastic Multipath Channel Modeling and Power Delay Profile Analysis for Terahertz-band Communication
}

\author{
Zahed Hossain* \\ University at Buffalo \\ Buffalo, New York \\ zahedhos@buffalo.edu
}

\author{
Carley Mollica \\ New Jersey Institute of Technology \\ Newark, New Jersey \\ crn5@njit.edu
}

\author{
Josep M. Jornet \\ University at Buffalo \\ Buffalo, New York \\ jmjornet@buffalo.edu
}

\begin{abstract}
Terahertz $(\mathrm{THz})$ band $(0.1-10 \mathrm{THz})$ communication is envisioned as a key technology to address the explosive growth in mobile data in recent years and the demand for high speed wireless communications. Major recent advances in device technologies are finally closing the $\mathrm{THz}$ gap and, thus, bringing this networking paradigm closer to reality. A general channel model is essential in designing and simulating communication techniques for the $\mathrm{THz}$ band. In this paper, we develop a stochastic multipath channel model for $\mathrm{THz}$ band communication for bounded area applications. Specifically, an analytical model for the number of single bounce multipath components and the power delay profile in a rectangular deployment scenario is derived, considering the density of obstacles, variable geometry of the rectangle, the signal blocking by obstacles and the propagation properties of $\mathrm{THz}$ signals. The model is independent of the physical layer technology and can be tailored to different application scenarios. Extensive simulation results are provided and utilized to validate the developed analytical model.
\end{abstract}

\section{CCS CONCEPTS}

- Mathematics of computing $\rightarrow$ Mathematical analysis; • Networks $\rightarrow$ Mobile ad hoc networks;

\section{KEYWORDS}

Terahertz-band Communication, Pulse-based Systems, Nanonetworks, Stochastic Multipath Channel Modeling

\section{ACM Reference format:}

Zahed Hossain, Carley Mollica, and Josep M. Jornet. 2017. Stochastic Multipath Channel Modeling and Power Delay Profile Analysis for Terahertz-band Communication. In Proceedings of NanoCom '17, Washington, DC, USA, September 27-29, 2017, 7 pages.

https://doi.org/10.1145/3109453.3109473

*Corresponding author.

Permission to make digital or hard copies of all or part of this work for personal or classroom use is granted without fee provided that copies are not made or distributed for profit or commercial advantage and that copies bear this notice and the full citation on the first page. Copyrights for components of this work owned by others than ACM must be honored. Abstracting with credit is permitted. To copy otherwise, or republish, to post on servers or to redistribute to lists, requires prior specific permission and/or a fee. Request permissions from permissions@acm.org.

NanoCom '17, September 27-29, 2017, Washington, DC, USA

() 2017 Association for Computing Machinery.

ACM ISBN 978-1-4503-4931-4/17/09. . \$15.00

https://doi.org/10.1145/3109453.3109473

\section{INTRODUCTION}

The way today's society creates, shares and consumes information has caused an explosive growth of wireless data traffic. This growth has been accompanied by an increasing demand for higher speed wireless communication anywhere, anytime. To support this demand, wireless data rate has doubled every eighteen months for the last three decades. Following this trend, wireless Terabit-per-second (Tbps) links are expected to become a reality within the next five years. In this context, Terahertz (THz)-band (0.1-10 THz) communication $[2,4,10]$ is envisioned as a key technology to satisfy the need for such very high data-rates, both in traditional networking paradigms as well as in novel nanoscale machine communication networks.

For many decades, the lack of compact high-power signal sources and high-sensitivity detectors able to work at room temperature has hampered the use of the $\mathrm{THz}$ band. However, many recent advancements with different technologies is finally closing the so-called $\mathrm{THz}$ gap. For example, on the one hand, in an electronic approach, III-V semiconductor technologies have demonstrated record performance in terms of output power, noise figure, and power-added efficiency at sub- $\mathrm{THz}$ frequencies, and are quickly approaching the $1 \mathrm{THz}[9,14]$. On the other hand, in an optics approach, Quantum Cascade Lasers are rising as potential candidates for high-power THz-band signal generation $[12,16]$. More recently, the use of nanomaterials such as graphene is enabling the development of plasmonic devices which intrinsically operate in the THz-band $[7,8]$.

Independently of the specific enabling technology, THzband communication brings many new opportunities to the wireless communication community. The THz band supports huge transmission bandwidths, which range from almost $10 \mathrm{THz}$ for distances below one meter, to multiple transmission windows, each tens to hundreds of $\mathrm{GHz}$ wide, for distances in the order of a few tens of meters. Nevertheless, this very large bandwidth comes at the cost of a very high propagation loss [6], mainly because of molecular absorption, which also creates a unique distance dependence on the available bandwidth. While the development of ultra-directional communication systems, such as those based on very large antenna array [1], will enable long-range $\mathrm{THz}$ communications, for the time being, THz-band communications are mainly constrained to very short (e.g., on-chip) and short (e.g., in a room) links.

In both application scenarios, an accurate channel model is needed to design and evaluate the performance of the 
communication system. Several channel models have been developed to date for the $\mathrm{THz}$ band. In [6], we developed the first channel model for line-of-sight (LOS) propagation over the entire $\mathrm{THz}$ band, by leveraging radiative transfer theory and revisiting the concept of molecular absorption. Some nonline-of-sight (NLOS) propagation models can also be found in the literature. These have been developed through extensive channel measurements $[13,15]$ or ray tracing simulations [5] in a fixed geometry environment. Both approaches are expensive and time consuming. In addition, these models are valid only for a particular deployment environment and need be developed again once the environment changes. In light of the random nature of wireless mobile networks, more general stochastic channel models are needed.

In this paper, we develop a stochastic multipath channel model for $\mathrm{THz}$ band communication for bounded area applications. Specifically, we derive analytical model for the number of single bounce multipath components and power delay profile in a rectangular deployment scenario. This general model is developed by taking into account the variable geometry of the rectangle, the density of reflecting objects in the area, the signal blocking by obstacles and the propagation properties of $\mathrm{THz}$ signals. The model is very general and is independent of the PHY layer technology. The model has been validated with extensive Monte Carlo numerical simulations in MATLAB.

The rest of the paper is organized as follows. In Section 2, we describe the system model. The stochastic model of multipath power delay profile is developed in Section 3. We validate our analytical model with numerical results in Section 4. Finally, we conclude the paper in Section 5.

\section{SYSTEM MODEL}

To model the multipath PDP, we need to take into account $\mathrm{THz}$ propagation characteristics, the geometry of the network and the spatial distribution of the nodes. In this section, we describe the system model utilized in the paper.

\subsection{THz propagation}

First, we describe how a $\mathrm{THz}$ signal is affected while propagating through the environment. The total path loss for the LOS component in the $\mathrm{THz}$ band consists molecular absorption loss and the spreading loss [6]. The path loss is given by

$$
P L_{L O S}(f, d)=\left(\frac{4 \pi f d}{c}\right)^{2} e^{k_{a b s}(f) d},
$$

where $f$ is the operating frequency in $H z, d$ is the separation between the transmitter and receiver, $c$ is the speed of light in free space and $k_{a b s}$ is frequency-dependent absorption coefficient of the medium [6]. For the NLOS components, we account for the reflection loss and the loss due to blockage of signals by intercepting objects. In this case, the total path loss is given by

$$
P L_{N L O S}(f, d)=\left(\frac{4 \pi f d}{c}\right)^{2} e^{k_{a b s}(f) d} \gamma(f),
$$

where $\gamma$ is the frequency-dependent reflection loss coefficient.

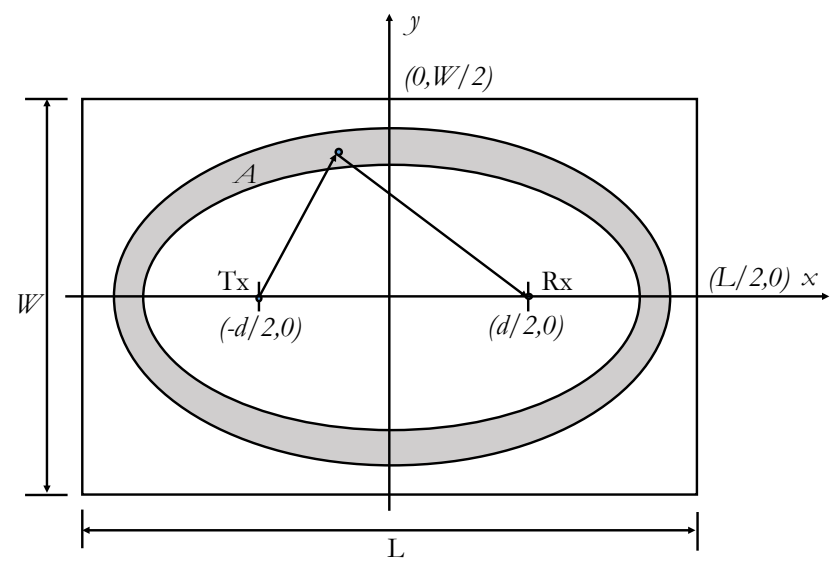

Figure 1: Geometry of the network considered.

\subsection{Network Topology}

We consider a rectangular area with length $L$ and width $W$. A rectangular area can be typical of an indoor network deployment or a network inside a chip. The origin of the coordinate system is assumed to be at the center of the rectangle. The transmitter and receiver are placed at equal distance $d / 2$ from the origin on the $x$ axis. We consider omnidirectional antennas are deployed at both the transmitter and the receiver. We also consider that different obstacles are randomly distributed inside the rectangle by following a spatial Poisson process with rate $\lambda$. These obstacles could be nanomachines in the nanoscale scenario or user devices with metallic components in the macroscale scenario. The probability of finding $k$ objects in the disc is given by

$$
P(k \in A)=\frac{(\lambda A)^{k}}{k !} e^{-\lambda A} .
$$

Under the Poisson assumption, the locations of the nodes follow independent and identically distributed uniform distributions. As a result, the signal transmitted by the transmitter will encounter multiple reflections and reach the receiver through different paths. These copies of the transmitted signal are called multipath components. In this paper, we consider only the single reflection components as the reflection loss and path loss are very high in the $\mathrm{THz}$ band. In addition, the strength of reflections from the walls of the rectangle are assumed to be minimal. If there is a LOS path between the transmitter and receiver, the first component will arrive with delay $\tau=\frac{d}{c}$.

As can be seen from Figure 1 and described in [11], all of the reflectors giving rise to single bounce components with delay between $\tau$ and $\tau+\Delta \tau$ lie within the region bounded by two ellipses. The major and minor radii of the inner ellipse are given as,

$$
a_{1}=\frac{c \tau}{2}, b_{1}=\sqrt{a_{1}^{2}-\left(\frac{d}{2}\right)^{2}}
$$


and the outer ellipse as

$$
a_{2}=\frac{c(\tau+\Delta \tau)}{2}, b_{2}=\sqrt{a_{2}^{2}-\left(\frac{d}{2}\right)^{2}} .
$$

In addition, the area of the shaded region is given as

$$
A=\pi\left(a_{2} b_{2}-a_{1} b_{1}\right) .
$$

The expected number of multipath components in the region with area $A$ is given by $\lambda A$. Hence, we can define a multipath density that gives the expected number of multipath components per unit of delay:

$$
f_{N}(\tau)=\lim _{\Delta \tau \rightarrow 0} \frac{\lambda A}{\Delta \tau} .
$$

From the density function, the number of multipath components within $\tau$ and $\tau+\Delta \tau$ can be found as follows:

$$
N(\tau)=\int_{0}^{\tau+\Delta \tau} f_{N}(\tau) d \tau-\int_{0}^{\tau} f_{N}(\tau) d \tau .
$$

The detailed derivation of $f_{N}(\tau)$ is the main contribution of this paper and is provided in Section 3. The $P D P(\tau)$ can now be readily found as follows

$$
P D P(\tau)=P L(f, c \tau) N(\tau)\left(1-P_{L O S}(d=c \tau)\right),
$$

$P_{L O S}$ is the distance-dependent probability that there exists a line of sight path at distance $d$.

\subsection{Physical and Link Layers}

The multipath channel model developed in this paper is independent of the PHY layer. In fact, the PDP derived can be utilized to characterize the channel and design the best PHY layer so that the received signal will suffer minimal inter symbol interference.

\section{MULTIPATH MODEL}

In this section, we analytically derive the number of multipath components and the LOS probability. However, due to the space limitation,we have omitted the detailed step-by-step process.

\subsection{Multipath Density}

The PDF of the number of NLOS paths is obtained by first replacing the values of $a_{1}, b_{1}, a_{2}, b_{2}$ in (7), which yields the following expression

$$
\begin{aligned}
& f_{N}(\tau)= \\
& \lim _{\Delta \tau \rightarrow 0} \frac{\lambda \pi\left(\frac{c(\tau+\Delta \tau)}{2} \sqrt{\frac{c^{2}(\tau+\Delta \tau)^{2}}{4}-\frac{d^{2}}{4}}-\frac{c \tau}{2} \sqrt{\frac{c^{2} \tau^{2}}{4}-\frac{d^{2}}{4}}\right)}{\Delta \tau},
\end{aligned}
$$

As $\Delta \tau$ approaches zero, the above expression takes on a 0/0 form. Applying L'Hospital's Rule, i.e., by taking the derivative of the numerator and the denominator with respect to $\Delta \tau$, and then applying the limit yields the following

$$
f_{N}(\tau)=\frac{\lambda \pi\left(2 c^{3} \tau^{2}-c d^{2}\right)}{4 \sqrt{c^{2} \tau^{2}-d^{2}}} .
$$

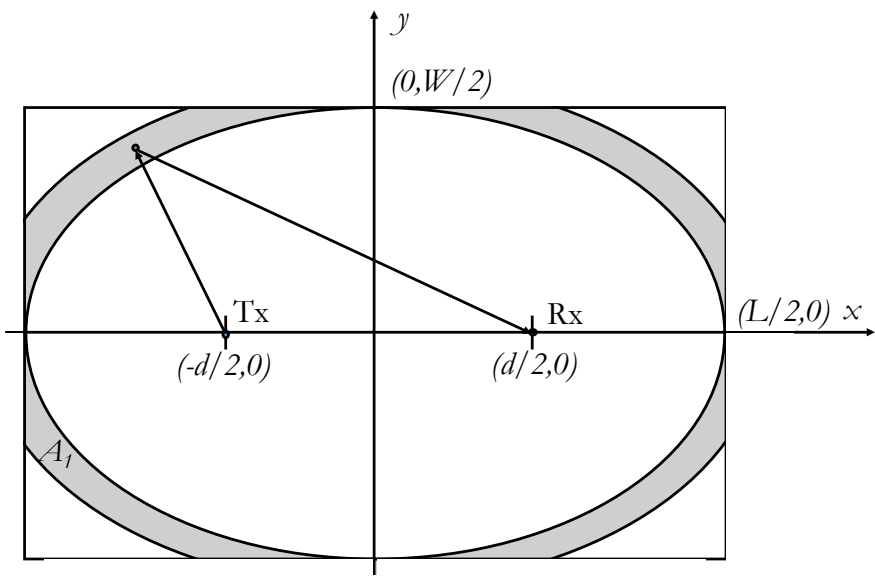

Figure 2: illustration of elliptical regions when $d$ equals critical distance.

As $\tau$ increases with the reflecting objects being farther away, the shaded region between the two ellipses also keeps growing. At a certain point, some part of the shaded region will fall outside the rectangle. Based on the distance between the transmitter and the receiver, the ellipses may pass the rectangle boundary on the major axis direction or minor axis direction or both directions at the same time. These three scenarios are illustrated below:

3.1.1 Scenario 1. The major and minor radii of the ellipse cross the wall at the same time for a critical distance $d_{\text {critical }}$, given by

$$
d_{\text {critical }}=\sqrt{L^{2}-W^{2}}
$$

This scenario is portrayed in Figure 2.

As can be seen from the figure, the ellipse touches the wall boundary on both sides at $\tau=\frac{L}{c}$. After that, the shaded region gets cut by the wall boundaries and form four equal size regions inside the rectangle. To obtain the multipath density in these regions, we need to find the area of the four regions. Since the four regions are identical, it is sufficient to find the area of the region in the first quadrant and multiply that by four. To find this area, we first find the intersecting points of the two ellipses with the line $y=\frac{W}{2}$. These points are given by

$$
x_{1}=\frac{a_{1}}{2 b_{1}} \sqrt{4 b_{1}^{2}-W^{2}}, x_{2}=\frac{a_{2}}{2 b_{2}} \sqrt{4 b_{2}^{2}-W^{2}}
$$

where $x_{1}$ is the intersection point between $y=\frac{W}{2}$ and the ellipse with delay $\tau$, and $x_{2}$ is the intersection point between $y=\frac{W}{2}$ and the ellipse with delay $\tau+\Delta \tau$. Hence, the area of the shaded region in the first quadrant can be calculated 
with the following integral,

$$
\begin{aligned}
A_{1} & =4\left(\int _ { x _ { 1 } } ^ { x _ { 2 } } \left(\frac{W}{2}-b_{1} \sqrt{\left.1-\frac{x^{2}}{a_{1}^{2}}\right) d x}\right.\right. \\
& \left.+\int_{x_{2}}^{\frac{L}{2}}\left(b_{2} \sqrt{1-\frac{x^{2}}{a_{2}^{2}}}-b_{1} \sqrt{1-\frac{x^{2}}{a_{1}^{2}}}\right) d x\right) .
\end{aligned}
$$

After carrying out the integral, the following expression is found for the total area inside the rectangle:

$$
\begin{aligned}
A_{1}= & \frac{a_{2} W}{8 b_{2}} \sqrt{4 b_{2}^{2}-W^{2}}-\frac{a_{1} W}{8 b_{1}} \sqrt{4 b_{1}^{2}-W^{2}} \\
& +\frac{a_{1} b_{1}}{2} \tan ^{-1} \frac{\sqrt{4 b_{1}^{2}-W^{2}}}{W}-\frac{a_{2} b_{2}}{2} \tan ^{-1} \frac{\sqrt{4 b_{2}^{2}-W^{2}}}{W} \\
& +\frac{a_{2} b_{2}}{2} \tan ^{-1} \frac{\frac{L}{2}}{\sqrt{a_{2}^{2}-\frac{L^{2}}{4}}}-\frac{a_{1} b_{1}}{2} \tan ^{-1} \frac{\frac{L}{2}}{\sqrt{a_{1}^{2}-\frac{L^{2}}{4}}} \\
& +\frac{L b_{2}}{4 a_{2}} \sqrt{a_{2}^{2}-\frac{L^{2}}{4}}-\frac{L b_{1}}{4 a_{1}} \sqrt{a_{1}^{2}-\frac{L^{2}}{4}}
\end{aligned}
$$

From (7), the multipath density for

$$
\frac{L}{c}<\tau<\frac{\sqrt{\left(\frac{L}{2}-\frac{d}{2}\right)^{2}+\frac{W^{2}}{4}}+\sqrt{\left(\frac{d}{2}+\frac{L}{2}\right)^{2}+\frac{W^{2}}{4}}}{c}
$$

is given by (17).

3.1.2 Scenario 2. As can be deduced from (4), when the distance is less than $d_{\text {critical }}$, the minor axis crosses the wall boundary first (see Figure 3). The minor axis crosses the boundary at $\tau=\frac{\sqrt{W^{2}+d^{2}}}{c}$. From the figure, it is easier to find the area of the shaded region by integrating with respect to $y$. In this case the area can be found by the following

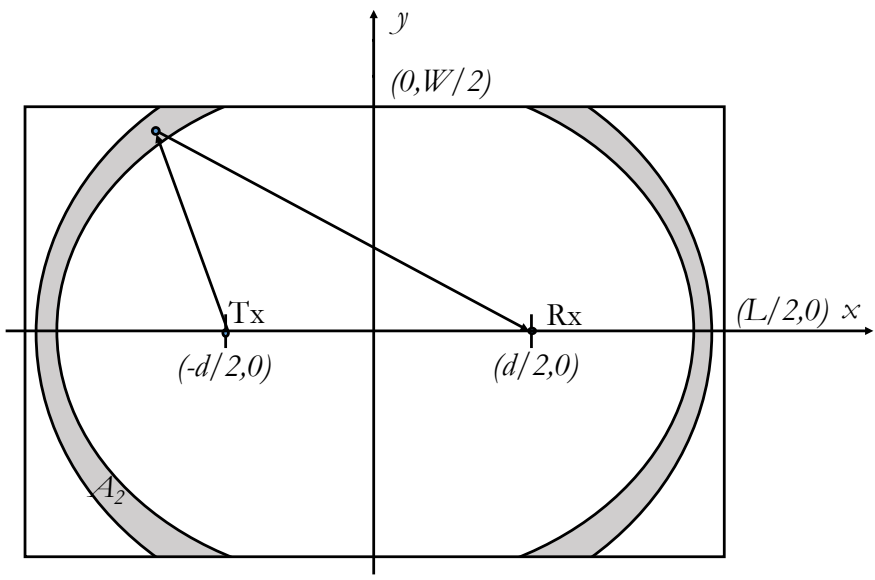

Figure 3: illustration of elliptical regions when $d$ is less than critical distance.

integral:

$$
A_{2}=4\left(\int_{0}^{\frac{W}{2}}\left(a_{2} \sqrt{1-\frac{y^{2}}{b_{2}^{2}}}-a_{1} \sqrt{1-\frac{y^{2}}{b_{1}^{2}}}\right) d y\right) .
$$

Evaluating the above integral yields the following expression,

$$
\begin{aligned}
A_{2}= & \frac{a_{2} W}{4 b_{2}} \sqrt{b_{2}^{2}-\frac{W^{2}}{4}}+\frac{a_{2} b_{2}}{2} \tan ^{-1} \frac{\frac{W}{2}}{\sqrt{b_{2}^{2}-\frac{W^{2}}{4}}} \\
& -\frac{a_{1} W}{4 b_{1}} \sqrt{b_{1}^{2}-\frac{W^{2}}{4}}-\frac{a_{1} b_{1}}{2} \tan ^{-1} \frac{\frac{W}{2}}{\sqrt{b_{1}^{2}-\frac{W^{2}}{4}}}
\end{aligned}
$$

This area formula is valid until $\tau=\frac{L}{c}$, when the major

$$
\begin{aligned}
f_{N 1}(\tau)= & \lambda\left(\frac{c L \sqrt{c^{2} \tau^{2}-d^{2}}}{16 \sqrt{\frac{c^{2} \tau^{2}}{4}-\frac{L^{2}}{4}}}+\frac{c L \sqrt{\frac{c^{2} \tau^{2}}{4}-\frac{L^{2}}{4}}}{4 \sqrt{c^{2} \tau^{2}-d^{2}}}-\frac{L \sqrt{c^{2} \tau^{2}-d^{2}} \sqrt{\frac{c^{2} \tau^{2}}{4}-\frac{L^{2}}{4}}}{4 c \tau^{2}}+\frac{1}{8} c \sqrt{c^{2} \tau^{2}-d^{2}} \tan ^{-1}\left(\frac{L}{2 \sqrt{\frac{c^{2} \tau^{2}}{4}-\frac{L^{2}}{4}}}\right)\right. \\
& +\frac{c W \sqrt{c^{2} \tau^{2}-d^{2}-W^{2}}}{8 \sqrt{c^{2} \tau^{2}-d^{2}}}-\frac{1}{8} c \sqrt{c^{2} \tau^{2}-d^{2}} \tan ^{-1}\left(\frac{\sqrt{c^{2} \tau^{2}-d^{2}-W^{2}}}{W}\right)-\frac{c^{3} L \tau^{2} \sqrt{c^{2} \tau^{2}-d^{2}}}{64\left(\frac{c^{2} \tau^{2}}{4}-\frac{L^{2}}{4}\right)^{3 / 2}\left(\frac{L^{2}}{4\left(\frac{c^{2} \tau^{2}}{4}-\frac{L^{2}}{4}\right)}+1\right)} \\
& +\frac{c^{3} \tau^{2} \tan ^{-1}\left(\frac{L}{2 \sqrt{\frac{c^{2} \tau^{2}}{4}-\frac{L^{2}}{4}}}\right)}{8 \sqrt{c^{2} \tau^{2}-d^{2}}}-\frac{c^{3} \tau^{2} W \sqrt{c^{2} \tau^{2}-d^{2}-W^{2}}}{8\left(c^{2} \tau^{2}-\frac{\left.d^{2}\right)^{3 / 2}}{8 \sqrt{c^{2} \tau^{2}-d^{2}} \sqrt{c^{2} \tau^{2}-d^{2}-W^{2}}}\right.} \\
& \left.-\frac{c^{3} \tau^{2} \sqrt{c^{2} \tau^{2}-d^{2}}}{8 W \sqrt{c^{2} \tau^{2}-d^{2}-W^{2}}\left(\frac{c^{2} \tau^{2}-d^{2}-W^{2}}{W^{2}}+1\right)}-\frac{c^{3} \tau^{2} W}{8 \sqrt{c^{2} \tau^{2}-d^{2}}}\right)
\end{aligned}
$$




$$
\begin{aligned}
& f_{N 2}(\tau)=\lambda\left(\frac{c W \sqrt{\frac{1}{4}\left(c^{2} \tau^{2}-d^{2}\right)-\frac{W^{2}}{4}}}{4 \sqrt{c^{2} \tau^{2}-d^{2}}}+\frac{1}{8} c \sqrt{c^{2} \tau^{2}-d^{2}} \tan ^{-1}\left(\frac{W}{2 \sqrt{\frac{1}{4}\left(c^{2} \tau^{2}-d^{2}\right)-\frac{W^{2}}{4}}}\right)-\frac{c^{3} \tau^{2} W \sqrt{\frac{1}{4}\left(c^{2} \tau^{2}-d^{2}\right)-\frac{W^{2}}{4}}}{4\left(c^{2} \tau^{2}-d^{2}\right)^{3 / 2}}\right. \\
& +\frac{c^{3} \tau^{2} W}{16 \sqrt{c^{2} \tau^{2}-d^{2}} \sqrt{\frac{1}{4}\left(c^{2} \tau^{2}-d^{2}\right)-\frac{W^{2}}{4}}}-\frac{c^{3} \tau^{2} W \sqrt{c^{2} \tau^{2}-d^{2}}}{64\left(\frac{1}{4}\left(c^{2} \tau^{2}-d^{2}\right)-\frac{W^{2}}{4}\right)^{3 / 2}\left(\frac{W^{2}}{4\left(\frac{1}{4}\left(c^{2} \tau^{2}-d^{2}\right)-\frac{W^{2}}{4}\right)}+1\right)} \\
& \left.\left.+\frac{c^{3} \tau^{2} \tan ^{-1}\left(\frac{W}{2 \sqrt{\frac{1}{4}\left(c^{2} \tau^{2}-d^{2}\right)-\frac{W^{2}}{4}}}\right)}{8 \sqrt{c^{2} \tau^{2}-d^{2}}}\right\}\right) \text {. }
\end{aligned}
$$

$$
\begin{aligned}
f_{N 3}(\tau)= & \lambda\left(\frac{c L \sqrt{c^{2} \tau^{2}-d^{2}}}{16 \sqrt{\frac{c^{2} \tau^{2}}{4}-\frac{L^{2}}{4}}}+\frac{c L \sqrt{\frac{c^{2} \tau^{2}}{4}-\frac{L^{2}}{4}}}{4 \sqrt{c^{2} \tau^{2}-d^{2}}}-\frac{L \sqrt{c^{2} \tau^{2}-d^{2}} \sqrt{\frac{c^{2} \tau^{2}}{4}-\frac{L^{2}}{4}}}{4 c \tau^{2}}+\frac{1}{8} c \sqrt{c^{2} \tau^{2}-d^{2}} \tan ^{-1}\left(\frac{L}{2 \sqrt{\frac{c^{2} \tau^{2}}{4}-\frac{L^{2}}{4}}}\right)\right. \\
& \left.-\frac{c^{3} L \tau^{2} \sqrt{c^{2} \tau^{2}-d^{2}}}{64\left(\frac{c^{2} \tau^{2}}{4}-\frac{L^{2}}{4}\right)^{3 / 2}\left(\frac{L^{2}}{4\left(\frac{c^{2} \tau^{2}}{4}-\frac{L^{2}}{4}\right)}+1\right)}+\frac{c^{3} \tau^{2} \tan ^{-1}\left(\frac{L}{2 \sqrt{\frac{c^{2} \tau^{2}}{4}-\frac{L^{2}}{4}}}\right)}{8 \sqrt{c^{2} \tau^{2}-d^{2}}}\right) .
\end{aligned}
$$

axis too crosses the wall boundary. From (7), the multipath density is now given by $(20)$.

For $\frac{L}{c}<\tau<\frac{\sqrt{\left(\frac{L}{2}-\frac{d}{2}\right)^{2}+\frac{W^{2}}{4}}+\sqrt{\left(\frac{d}{2}+\frac{L}{2}\right)^{2}+\frac{W^{2}}{4}}}{c}$, the area is given by (15) and the multipath density function is given by (17).

3.1.3 Scenario 3. If the distance is greater than $d_{\text {critical }}$, then the major axis crosses the wall boundary first (see Figure 4). The major axis crosses the boundary at $\tau=\frac{L}{c}$.

In this case the area of the shaded region that is inside the rectangle is given by:

$$
\begin{aligned}
A_{3} & =4\left(\int_{0}^{\frac{L}{2}}\left(b_{2} \sqrt{1-\frac{x^{2}}{a_{2}^{2}}}-b_{1} \sqrt{\left.1-\frac{x^{2}}{a_{1}^{2}}\right) d x}\right)\right. \\
& =\frac{b_{2} L}{4 a_{2}} \sqrt{a_{2}^{2}-\frac{L^{2}}{4}}+\frac{a_{2} b_{2}}{2} \tan ^{-1} \frac{\frac{L}{2}}{\sqrt{a_{2}^{2}-\frac{L^{2}}{4}}} \\
& -\frac{b_{1} L}{4 a_{1}} \sqrt{a_{1}^{2}-\frac{L^{2}}{4}}-\frac{a_{1} b_{1}}{2} \tan ^{-1} \frac{\frac{L}{2}}{\sqrt{a_{1}^{2}-\frac{L^{2}}{4}}} .
\end{aligned}
$$

This area formula is valid until $\tau=\frac{\sqrt{W^{2}+d^{2}}}{c}$, when the major axis too crosses the wall boundary. Applying the definition in (7), the multipath density is now given by (21). For $\frac{\sqrt{W^{2}+d^{2}}}{c}<\tau<\frac{\sqrt{\left(\frac{L}{2}-\frac{d}{2}\right)^{2}+\frac{W^{2}}{4}}+\sqrt{\left(\frac{d}{2}+\frac{L}{2}\right)^{2}+\frac{W^{2}}{4}}}{c}$, the area is given by (15) and the multipath density function is given by $(17)$.

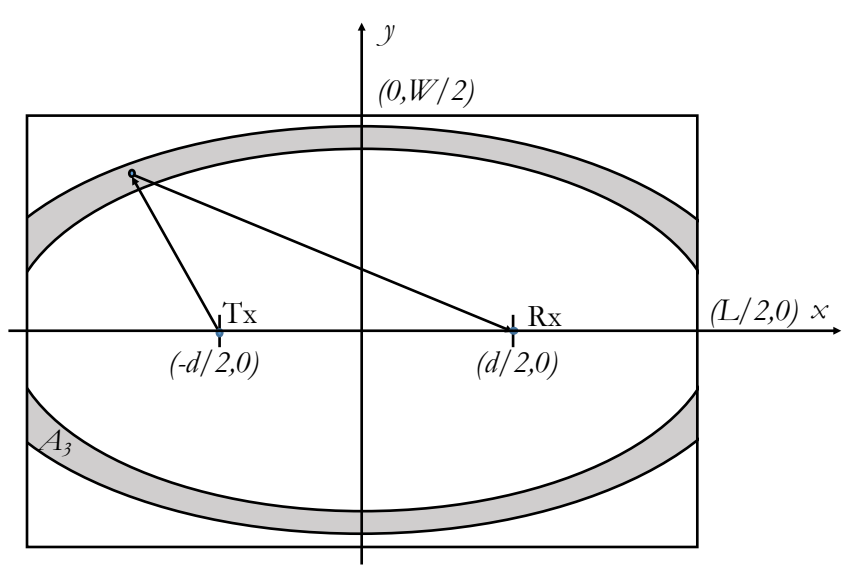

Figure 4: illustration of elliptical regions when $d$ is greater than critical distance.

\subsection{LOS Probability}

As the electromagnetic signals in $\mathrm{THz}$ band can not penetrate the obstacles, we need to take into account that a multipath component might get blocked on the way. To calculate the blocking probability, we consider that the obstacles are of circular shape with radius $r$ and their centers follow the same Poisson process described in 2. We first calculate the LOS probability following a similar approach used in [3]. As can be seen from Figure 5, the link between the transmitter and receiver is blocked if the center of any of the circular obstacles falls inside the shaded region. The number of cir- 


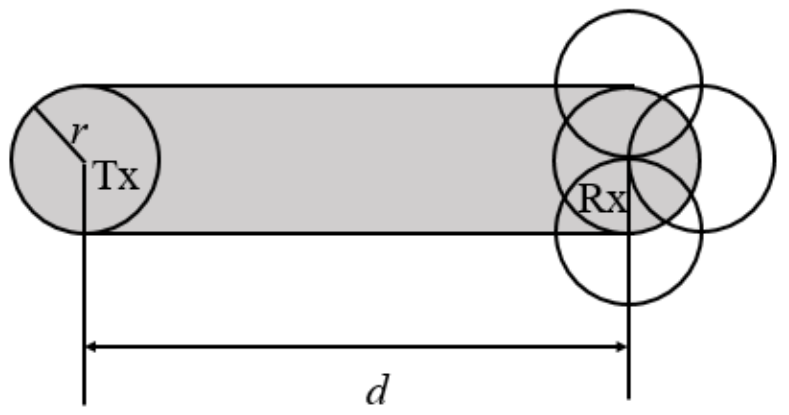

Figure 5: Blocking region between two objects with separation $d$.

cular obstacles whose centers are inside the shaded region is a Poisson random variable with mean $\lambda A_{\text {blockage }}$, where $A_{\text {blockage }}$ is the area of the shaded region. A blockage can be calculated easily by dividing the shaded regions into three regions, namely a half circle of radius $r$, a rectangle of width $r$ and length $d$ and another half circle of radius $r$.

$$
A_{\text {blockage }}=r d+\pi r^{2} \text {. }
$$

The LOS probability that no obstacle crosses the link between the transmitter and the receiver is a distance-dependent function given by

$$
\begin{aligned}
P_{L O S}(d) & =P\left(0 \in A_{\text {blockage }}\right)=\frac{\left(\lambda A_{\text {blockage }}\right)^{0}}{0 !} e^{-\lambda A_{\text {blockage }}} \\
& =e^{-\lambda\left(r d+\pi r^{2}\right)} .
\end{aligned}
$$

\section{NUMERICAL RESULTS}

In this section we validate our analytical results through Monte Carlo simulation in MATLAB. A rectangular area of length $3 \mathrm{~m}$ and width $2 \mathrm{~m}$ has been used to simulate the multipath propagation environment. A collection of circular shaped obstacles with radius $5 \mathrm{~cm}$ has been uniformly distributed in the area with a density of $\lambda=100,150$ and 200 nodes $/ \mathrm{m}^{2}$. The operating frequency $f=1.0345 \mathrm{THz}$ was chosen as the center frequency of the first absorption-defined $\mathrm{THz}$ transmission window [6] and the corresponding absorption coefficient $k_{a b s}=3.0155 \times 10^{-2} \mathrm{~m}^{-1}$ was assumed to be constant over the whole window. The reflection loss coefficient $\gamma$ was assumed to be unity as most of the devices behave as perfect reflectors for $\mathrm{THz}$ signals, but the model remains valid for any value of $\gamma$. The final results were found by averaging over a total of 1000 realizations of the system. A histogram of the number of multipath components was generated using a bin size of $\Delta \tau=10^{-11} \mathrm{~s}$ for three distances corresponding to the three cases used in the analytical model. Figure 6 depicts the case where separation between the transmitter and the receiver equals the critical distance of $d_{\text {critical }}=\sqrt{5} \mathrm{~m}$. As can be seen from the figure, the analytical model matches very closely with the simulation results. Note also that there are two regions in this case since the both of the radii of the ellipse crosses the wall boundary at the same time. Figure 7 and Figure 8 show the other two

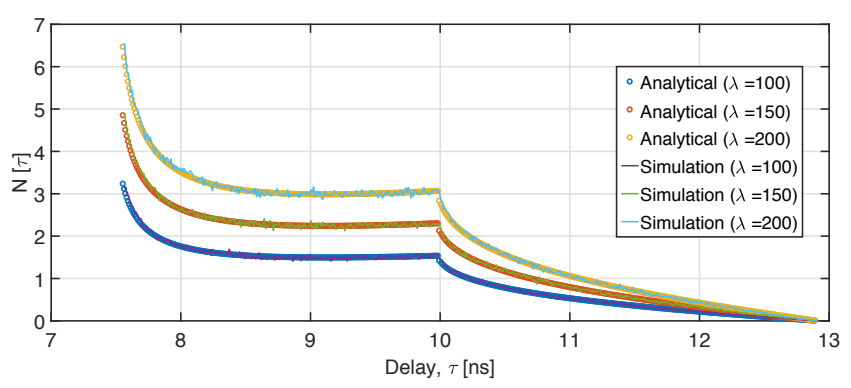

Figure 6: Number of multipath components as function of path delay when $d$ is equal to $d_{\text {critical }}$.

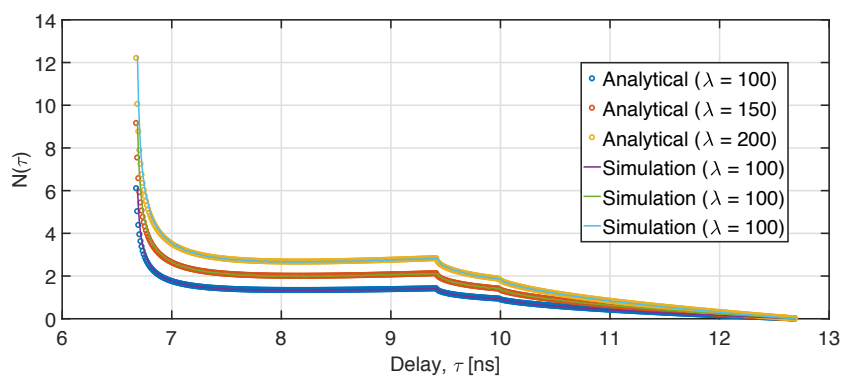

Figure 7: Number of multipath components as function of path delay when $d$ is less than $d_{\text {critical }}$.

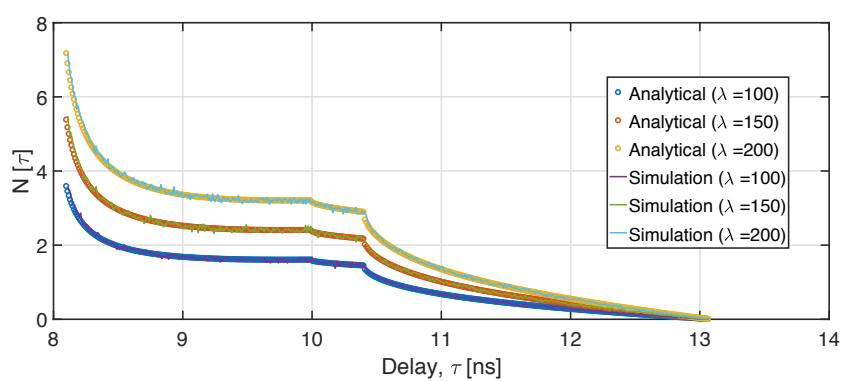

Figure 8: Number of multipath components as function of path delay when $d$ is greater than $d_{\text {critical }}$.

cases when the Tx-Rx separation is $\operatorname{less}(d=2 m)$ than and greater $(d=2.4 \mathrm{~m})$ than the critical distance respectively. In these cases, there are three regions due to one of the radii crossing the wall boundary first. The multipath power delay profile has been plotted in Figure 9 for the Tx-Rx separation equal to the critical distance. As can be seen from the figure, the power loss of the multipath components increases with increasing density of obstacles. This behavior is expected from the exponential decay of the LOS probability in (24) with the density of obstacles. 


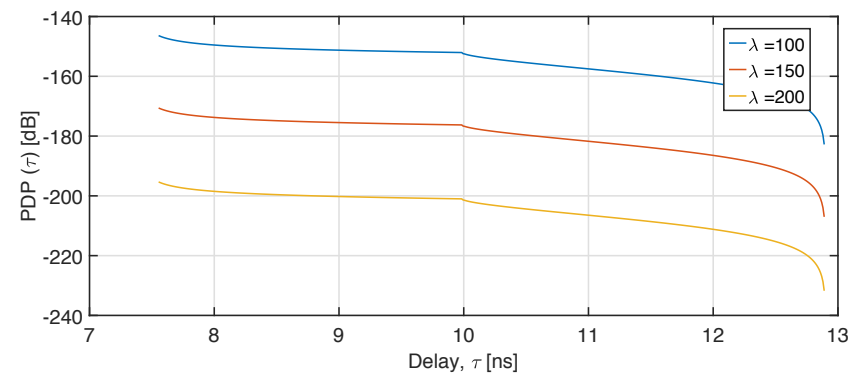

Figure 9: Multipath PDP as a function of $\tau$ when Tx-Rx separation is $d_{\text {critical }}$.

\section{CONCLUSION}

In this paper, a stochastic multipath channel model for $\mathrm{THz}-$ band communication network has been developed, by considering an enclosed geometry in light of expected applications of $\mathrm{THz}$ networks. The model is general in the sense that it is independent of the PHY layer. First, the number of multipath components at a certain delay has been analytically derived using the density of reflecting objects and the sides of the rectangle as model parameters. Then the LOS probability has been formulated by taking into account that the reflecting points can also block the multipath components. Finally, the multipath PDP has been found by multiplying the number of multipath components with path loss associated with the corresponding distance. The analytical model has been validated by means of extensive simulations with MATLAB. As part of our future work, we will extend these results to account for directional antennas needed for longer communication distances (e.g., larger rooms).

\section{ACKNOWLEDGMENTS}

This work was partially supported by the Air Force Office of Scientific Research (AFOSR) under Grant FA9550-16-1-0188.

\section{REFERENCES}

[1] I. F. Akyildiz and J. M. Jornet. Realizing ultra-massive MIMO $(1024 \times 1024)$ communication in the $(0.06-10)$ terahertz band
Nano Communication Networks, 8:46-54, 2016.

[2] I. F. Akyildiz, J. M. Jornet, and C. Han. Terahertz band: Next frontier for wireless communications. Physical Communication (Elsevier) Journal, 12:16 - 32, Sept. 2014.

[3] T. Bai, R. Vaze, and R. W. Heath. Analysis of blockage effects on urban cellular networks. IEEE Transactions on Wireless Communications, 13(9):5070-5083, 2014.

[4] J. Federici and L. Moeller. Review of terahertz and subterahertz wireless communications. Journal of Applied Physics, 107(11):111101, 2010.

[5] C. Han, A. O. Bicen, and I. Akyildiz. Multi-ray channel modeling and wideband characterization for wireless communications in the terahertz band. IEEE Transactions on Wireless Communications, 14(5):2402-2412, May 2015.

[6] J. M. Jornet and I. F. Akyildiz. Channel modeling and capacity analysis of electromagnetic wireless nanonetworks in the terahertz band. IEEE Transactions on Wireless Communications, 10(10):3211-3221, Oct. 2011.

[7] J. M. Jornet and I. F. Akyildiz. Graphene-based plasmonic nanoantenna for terahertz band communication in nanonetworks. IEEE JSAC, Special Issue on Emerging Technologies for Communications, 12(12):685-694, Dec. 2013

[8] J. M. Jornet and I. F. Akyildiz. Graphene-based plasmonic nanotransceiver for terahertz band communication. In Proc. of European Conference on Antennas and Propagation (EuCAP), 2014.

[9] Y. Kurita, G. Ducournau, D. Coquillat, A. Satou, K. Kobayashi, S. B. Tombet, Y. Meziani, V. Popov, W. Knap, T. Suemitsu, et al. Ultrahigh sensitive sub-terahertz detection by InP-based asymmetric dual-grating-gate high-electron-mobility transistors and their broadband characteristics. Applied Physics Letters, 104(25):251114, 2014.

[10] T. Kurner and S. Priebe. Towards THz Communications-Status in Research, Standardization and Regulation. Journal of Infrared, Millimeter, and Terahertz Waves, 35(1):53-62, 2014.

[11] J. Liberti and T. Rappaport. A geometrically based model for line-of-sight multipath radio channels. In Proc of IEEE Vehicular Technology Conference, pages 844-848, 1996.

[12] Q. Lu, D. Wu, S. Sengupta, S. Slivken, and M. Razeghi. Room temperature continuous wave, monolithic tunable $\mathrm{THz}$ sources based on highly efficient mid-infrared quantum cascade lasers. Scientific reports, 6, 2016.

[13] S. Priebe and T. Kurner. Stochastic modeling of thz indoor radio channels. IEEE Transactions on Wireless Communications, 12(9):4445-4455, 2013.

[14] V. Radisic, K. Leong, D. Scott, C. Monier, X. Mei, W. Deal, and A. Gutierrez-Aitken. Sub-millimeter wave inp technologies and integration techniques. In IEEE MTT-S International Microwave Symposium (IMS), pages 1-4, May 2015.

[15] A. Saleh and R. Valenzuela. A statistical model for indoor multipath propagation. IEEE Journal on selected areas in communications, 5(2):128-137, 1987.

[16] S. Slivken and M. Razeghi. High power, electrically tunable quantum cascade lasers. In SPIE OPTO, pages $97550 \mathrm{C}-97550 \mathrm{C}$. International Society for Optics and Photonics, 2016. 